\section{In-stent Restenosis after Drug-eluting Stent Implantation in Rheumatoid Arthritis: Possible Protective Effect of Methotrexate}

\section{To the Editor:}

Patients with rheumatoid arthritis (RA) are at higher risk of cardiovascular events compared with the general population ${ }^{1}$. Therefore, these patients with RA have a greater chance of undergoing percutaneous coronary intervention (PCI). In-stent restenosis (ISR) after PCI results from damage to arteries with subsequent neointimal tissue proliferation ${ }^{2}$ and is considered the most significant problem in coronary interventional treatment. Compared with bare-metal stents (BMS), drug-eluting stents (DES) have dramatically reduced the rate of $\mathrm{ISR}^{3}$. Nevertheless, ISR after DES still exists, with an occurrence of $3-20 \%$.
Methotrexate (MTX), the most important drug used in RA, is a folate antagonist that blocks the $\mathrm{S}$ phase of cell division and consequently blocks mitosis ${ }^{4}$. An intravenous injection of MTX resulted in the reduction of in-stent neoatherosclerosis in an animal model ${ }^{5}$. Further, a clinical study suggested the positive effect of oral MTX on ISR after BMS implantation ${ }^{6}$. However, little is known about the effect of oral MTX on ISR after DES implantation. Therefore, we aimed to assess whether the oral administration of MTX in patients with RA has a beneficial effect in preventing ISR after DES implantation.

We retrospectively reviewed patients with RA who underwent DES implantation at a tertiary referral hospital in Seoul, South Korea, between January 2005 and March 2017. All patients fulfilled the 1987 American College of Rheumatology criteria for the classification of RA. The established risk factors [young age, diabetes mellitus, multivessel disease, ostial

Table 1. Comparison between treated vessels in the MTX group and non-MTX group. Values are n (\%) unless otherwise specified.

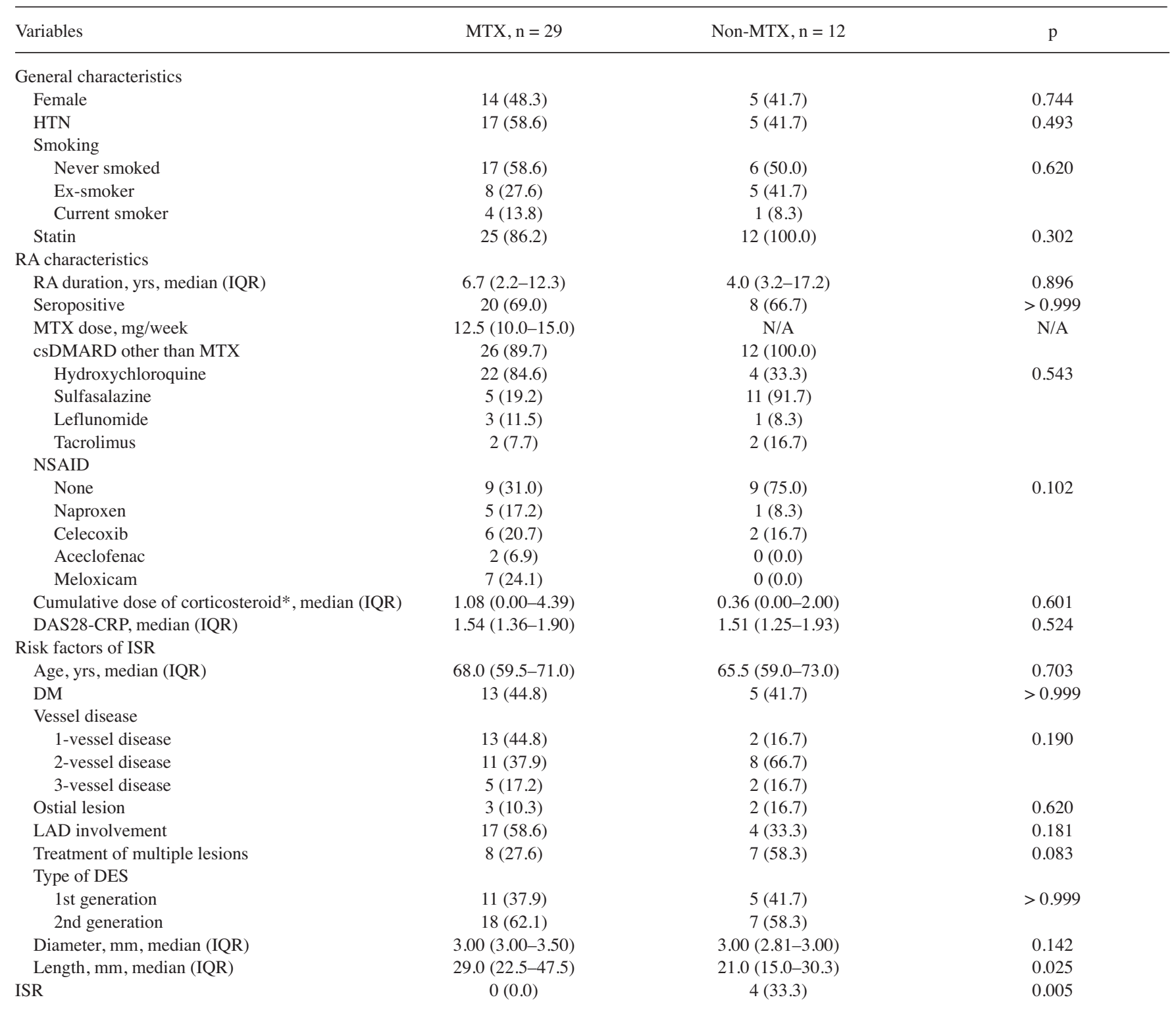

* g of prednisolone or its equivalent. MTX: methotrexate; HTN: hypertension; RA: rheumatoid arthritis; IQR: interquartile range; NSAID: nonsteroidal antiinflammatory drug; csDMARD: conventional synthetic disease-modifying antirheumatic drug; DAS28-CRP: 28-joint count Disease Activity Score using C-reactive protein; ISR: in-stent restenosis; DM: diabetes mellitus; LAD: left anterior descending artery; DES: drug-eluting stent; N/A: not applicable. 
lesion, treatment of multiple lesions, type of DES, longer physical length of stent, small reference diameter, and non-left anterior descending artery (LAD) lesions] $]^{7,8,9}$ for ISR after DES and ISR incidence were compared between the MTX group and non-MTX group. MTX group was defined as patients who had ever used MTX after DES implantation. To exclude the possible confounding effect of hydroxychloroquine (HCQ), which is known to have a cardioprotective effect, we also compared the MTX group and non-MTX group among the HCQ users (subgroup analysis). This study was approved by the Institutional Review Board (Asan Medical Center, IRB No. 2017-0919)

Mann-Whitney U test and Fisher's exact test were performed to compare continuous and categorical variables, respectively. Cox proportional hazard model was performed to evaluate whether MTX is associated with risk of developing ISR. Because MTX is a time-varying variable, cumulative dose of MTX was used for this analysis. Survival rates were analyzed using the Kaplan-Meier method and compared using the log-rank test.

A total of 29 patients with RA underwent PCI. Forty-one vessel lesions were treated by DES implantation. The median age was 67 years, and $44.8 \%$ were female. The most commonly used disease-modifying antirheumatic drug was MTX [21 (72.4\%)], followed by HCQ and sulfasalazine. Biologic agent was used in 1 patient (etanercept, 3.4\%).

Among the 21 patients receiving MTX, there were 29 treated vessels. Further, there were 12 treated vessels in the 8 patients not receiving MTX. A comparison between the 2 groups is shown in Table 1. Established predictors of ISR did not differ between the 2 groups, except for the longer stented length in the MTX group [median 29.0 (IQR 22.5-47.5) vs 21.0 $(15.0-30.3), p=0.025]$. Nevertheless, the incidence of ISR was significantly lower in the MTX group than in the non-MTX group [0 (0\%) vs $4(33.3 \%)$, $\mathrm{p}=0.005]$. In the subgroup analysis of HCQ users, risk factors of ISR did not differ between the MTX group and non-MTX group. Incidence of ISR remained lower in the MTX group [0 $(0 \%)$ vs $1(25.0 \%), \mathrm{p}=0.017$; Supplementary Table 1, available with the online version of this letter]. On Cox proportional hazard model, cumulative dose of MTX was associated with decreased risk of developing ISR (HR $0.174,95 \%$ CI $0.032-0.940$, $p=0.042$ ). Kaplan-Meier curves showed a significantly higher rate of ISR in the treated vessels in the non-MTX group than in the MTX group $(p=0.001 ;$ Figure 1). The median time from DES implantation to ISR in the non-MTX group was 106.8 (81.1-109.0) months, which is "late" restenosis. Considering that there was no occurrence of ISR during the longterm followup in the MTX group, MTX might be beneficial in the late restenosis after DES implantation.

Neointimal hyperplasia is the primary component of restenosis after stent deployment. When vascular injury occurs, inflammatory cells and proinflammatory cytokines lead to the proliferation and migration of vascular smooth muscle cells within the media and intima, resulting in restenosis ${ }^{10}$. MTX has both antiproliferative and antiinflammatory properties ${ }^{4}$. Considering the pathogenesis of ISR, antiproliferative and antiinflammatory effects of MTX might have contributed in suppressing neointimal formation.

Based on our real-world results, we have shown that administration of oral MTX in patients with RA was associated with lower incidence of ISR after DES implantation. Because the study population was too small and multiple vessels from the same patients were counted, which might be a potential source of bias, further studies are needed to confirm the present finding in larger sample sizes

OH CHAN KWON, MD, Division of Rheumatology, Department of Medicine; JI SEON OH, MD, PhD, Clinical Research Center; YONG-GIL KIM, MD, PhD, Division of Rheumatology, Department of Medicine, University of Ulsan, College of Medicine, Asan Medical Center, Seoul, South Korea. Supported by a grant (2017-655) from the Asan Institute for Life Sciences, Asan Medical Center, Korea. Address correspondence to Dr. Y.G. Kim, Division of Rheumatology, University of Ulsan, College of Medicine, Asan Medical Center, 88 Olympic-ro 43-gil, Songpa-gu, Seoul 05505, South Korea. E-mail: bestmd2000@amc.seoul.kr

\section{ONLINE SUPPLEMENT}

Supplementary material accompanies the online version of this letter.

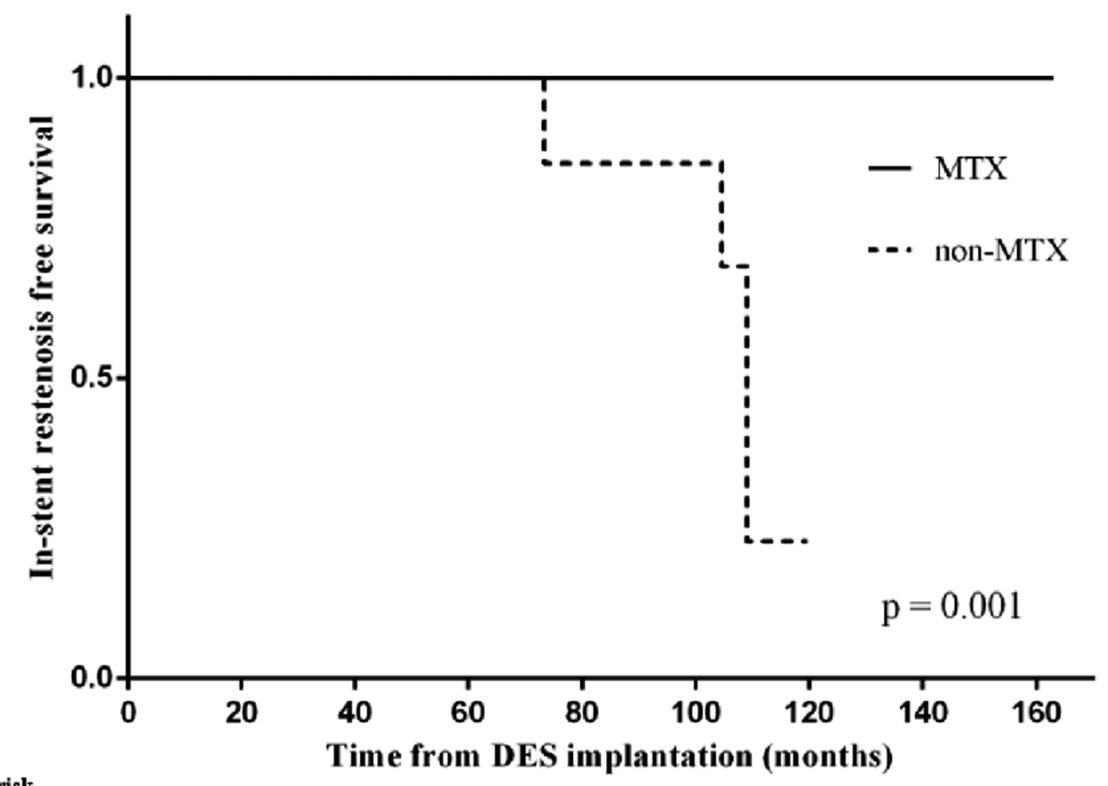

Number at risk

$\begin{array}{lccccccccc}\text { MTX group } & 29 & 23 & 18 & 16 & 14 & 12 & 7 & 5 & 1 \\ \text { non-MTX group } & 12 & 10 & 9 & 8 & 7 & 6 & 1 & 0 & 0\end{array}$

Figure 1. Kaplan-Meier survival curves for in-stent restenosis between MTX-treated (MTX group) and nonMTX groups. MTX: methotrexate; DES: drug-eluting stents. 


\section{REFERENCES}

1. Avina-Zubieta JA, Thomas J, Sadatsafavi M, Lehman AJ, Lacaille D. Risk of incident cardiovascular events in patients with rheumatoid arthritis: a meta-analysis of observational studies. Ann Rheum Dis 2012;71:1524-9.

2. Dangas GD, Claessen BE, Caixeta A, Sanidas EA, Mintz GS, Mehran R. In-stent restenosis in the drug-eluting stent era. J Am Coll Cardiol 2010;56:1897-907.

3. Stettler C, Wandel S, Allemann S, Kastrati A, Morice MC, Schomig A, et al. Outcomes associated with drug-eluting and bare-metal stents: a collaborative network meta-analysis. Lancet 2007; 370:937-48.

4. Tunali-Akbay T, Sehirli O, Ercan F, Sener G. Resveratrol protects against methotrexate-induced hepatic injury in rats. J Pharm Pharm Sci 2010;13:303-10.

5. Zhang R, Chen S, Zhang H, Liu Q, Xing J, Zhao Q, et al. Effects of methotrexate in a rabbit model of in-stent neoatherosclerosis: an optical coherence tomography study. Sci Rep 2016;6:33657.

6. Gouveia V, Oliveira DC, Tenorio E, Brito N, Sarinho E. Percutaneous coronary intervention: safety of methotrexate and its possible benefits on restenosis after bare-metal stent deployment. Cardiol Res 2016;7:104-9.
7. Lemos PA, Hoye A, Goedhart D, Arampatzis CA, Saia F, van der Giessen WJ, et al. Clinical, angiographic, and procedural predictors of angiographic restenosis after sirolimus-eluting stent implantation in complex patients: an evaluation from the Rapamycin-Eluting Stent Evaluated At Rotterdam Cardiology Hospital (RESEARCH) study. Circulation 2004;109:1366-70.

8. Kastrati A, Dibra A, Mehilli J, Mayer S, Pinieck S, Pache J, et al. Predictive factors of restenosis after coronary implantation of sirolimus- or paclitaxel-eluting stents. Circulation 2006; 113:2293-300.

9. Zahn R, Hamm CW, Schneider S, Zeymer U, Nienaber CA, Richardt G, et al; German Cypher Stent Registry. Incidence and predictors of target vessel revascularization and clinical event rates of the sirolimus-eluting coronary stent (results from the prospective multicenter German Cypher Stent Registry). Am J Cardiol 2005;95:1302-8.

10. Chaabane C, Otsuka F, Virmani R, Bochaton-Piallat ML. Biological responses in stented arteries. Cardiovasc Res 2013;99:353-63.

J Rheumatol First Release August 1 2018; doi:10.3899/jrheum.180171 